AperTO - Archivio Istituzionale Open Access dell'Università di Torino

\title{
Mind the red thread! 3D metaphors for cultural heritage visualization
}

\section{This is the author's manuscript}

Original Citation:

Availability:

This version is available http://hdl.handle.net/2318/1577993

since 2016-11-29T15:41:00Z

Publisher:

Institute of Electrical and Electronics Engineers Inc.

Published version:

DOI:10.1109/DigitalHeritage.2015.7419522

Terms of use:

Open Access

Anyone can freely access the full text of works made available as "Open Access". Works made available under a Creative Commons license can be used according to the terms and conditions of said license. Use of all other works requires consent of the right holder (author or publisher) if not exempted from copyright protection by the applicable law. 
This is the author's final version of the contribution published as:

Damiano, Rossana; Lombardo, Vincenzo; Lieto, Antonio; Borra, Davide. Mind the red thread! 3D metaphors for cultural heritage visualization, in: 2015 Digital Heritage International Congress, Digital Heritage 2015, Institute of Electrical and Electronics Engineers Inc., 2015, 9781509000487, pp: 355-358.

The publisher's version is available at:

http://xplorestaging.ieee.org/ielx7/7406203/7419433/7419522.pdf?arnumber=7419522

When citing, please refer to the published version.

Link to this full text:

http://hdl.handle.net/2318/1577993 


\section{Mind the Red Thread! 3D Metaphors for Cultural Heritage Visualization}

\author{
Rossana Damiano and Vincenzo Lombardo \\ Dipartimento di Informatica and CIRMA \\ Università di Torino, Italy
}

\begin{abstract}
In cultural heritage, the current shift toward semantic encoding opens the way to interfaces that provide a visual representation of the complex relations among heritage artifacts. In this paper we describe Labyrinth $3 D$, a virtual environment where the user can navigate the semantic relations among the items contained in a repository of artworks.
\end{abstract}

Keywords-3D visualization, cultural heritage, ontologies

\section{INTRODUCTION}

The semantic annotation of heritage objects opens the way to applications that leverage the description of their properties and relations to support the user's access [1], [2], [3]. A major shortcoming of this approach is that the semantic representation, in most cases, cannot be exposed directly to the users, since it is encoded in a machine readable form oriented to interoperability and formal reasoning. System designers, then, must devise metaphors through which the semantically encoded conceptual models underlying cultural heritage can be conveyed to the users, so that these models can effectively improve the interaction between the users and the archives.

This paper describes a 3D spatial layout for the exploration of cultural heritage repositories where game elements co-exist with the paradigm of exploratory search [4]. Our vision leverages the use of visual technologies, widely employed in real world applications that range from video games to edutainment and installation art, to create an immersive user experience. We adapt the paradigm of virtual tours of heritage locations to the exploration of heritage repositories, proposing a virtual environment that the users can explore to navigate the contents of a repository. The cues of the exploration are the visual and spatial metaphors (e.g., aspect, proximity, etc.) encoded in the virtual space, with the causal and associative relations over the contents represented by a maze of physical connections. In order to exemplify this approach, we describe a 3D application that relies on an ontology of "cultural archetypes" to create and visualize a "labyrinth" of semantic relations over the artworks contained in a repository. ${ }^{1}$

The plan of the paper is the following: after briefly surveying the related work (Section II), in Section III we provide an overview of the system and describe the user experience through a navigation example. Section IV illustrates the system architecture and the implementation of the 3D generation of the virtual environment. Conclusion ends the paper.

\footnotetext{
${ }^{1}$ This work was partially supported by Regione Piemonte, POR-FESR 2007 13, Polo di Innovazione per la Creatività Digitale e la Multimedialità.
}

\author{
Antonio Lieto \\ Dipartimento di Informatica \\ Università di Torino, Italy
}

\author{
Davide Borra \\ NoReal \\ Torino, Italy
}

\section{RELATED WORK}

In cultural heritage, the metaphor of the archive is still adopted by most systems to support the access to contents, with keyword-based search and content indexing as the main tools provided to the users. As pointed out by [5], however, the general public is not committed to specific search goals, that can be expressed through a formal query: rather, they are normally interested in exploring the archive in search for items that catch their attention. So, paradigms that support exploratory search are required to meet the needs of this audience, which has acquired a growing importance in the age of mass digitalization. In the standard case, then, the output of a query returns a list of objects (books, pictures, videos, etc.), with no explicit representation of the relations they have with the input keywords, let alone the connections among them. The visualization framework we describe in this paper aims at innovating the access to cultural heritage archives: our proposal translates the information about cultural heritage objects, expressed in a semantic format, into an executable visual representation where the relations over the objects are mapped onto a 3D environment. This approach stems from the observations in favor of $3 \mathrm{D}$ virtual environments collected in interdisciplinary studies. On the one side, the use of visual metaphors allows conveying conceptual models in an immediate way (cf. the established line of research in information visualization [6]); on the other side, the use of $3 \mathrm{D}$ is also known as a way to promote engagement in the transmission of knowledge [7]. In particular, [8] show that the use of the game in tandem with visual metaphors results in increased levels of learning in educational applications.

In cultural heritage, the use of 3D visualization is normally intended as a support for dissemination and research. Dissemination encompasses both virtual equivalents of physically existing locations, such as museums and historical buildings, and reconstructions of environments that have disappeared. The project Rome Reborn [9] relies on 3D to let the user experience the city of Rome in the IV century, providing an example of the use of 3D to reconstruct ancient locations; the VEP project [10], a reconstruction of the Poème Electronique Virtuel created by Varèse and Le Corbusier in 1958 at the Brussels World Fair (1958) and subsequently dismantled, relies on 3D to address the impermanence of today's artifacts. Finally, some recent projects employ 3D visualization to support research activities, creating virtual environments for training and collaboration [11], [12].

The work we present in this paper differentiates from the approaches surveyed above since the 3D representation 


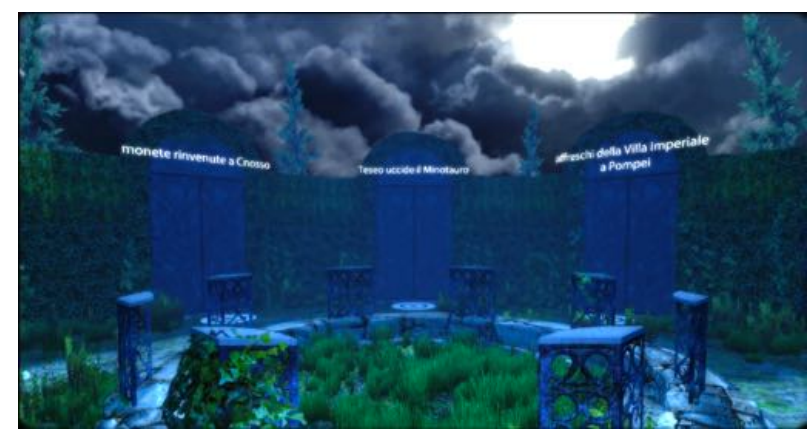

Fig. 1. A node of the labyrinth.

is not aimed at reconstructing a real environment: rather, the virtual environment is employed a tool to convey the semantic relations over a set of cultural objects through a visual layout.

\section{USER EXPERIENCE}

Targeted at the general audience, Labyrinth 3D is aimed at encouraging the users to explore a cultural heritage repository in an immersive, engaging way, as a way to promote heritage dissemination. The user is immersed in a 3D labyrinth where the physical connections between the items correspond to meaning relations between them. Turn after turn, the user decides where to address her/his exploration, based on her/his curiosity, in a serendipic discovery of new items. The user experience trades off the users' need for orientation, achieved through the meaning relations among the displayed items, and the unpredictability of an unknown, open environment.

The design of the $3 \mathrm{D}$ environment is inspired by the metaphor of the labyrinth, an archetype deeply rooted in Western Culture, as witnessed by several archaeological locations across Europe [13]. The metaphor of the labyrinth was chosen because it lends itself to convey the graph like nature of the relations among the cultural heritage objects, guaranteeing at the same time the immediacy of use. Formally, the labyrinth consists of a graph of nodes [14], a structure that is easily mapped onto a set of objects (labyrinth nodes) and relations over them (pathways connecting the nodes). The screenshot in Fig. 1 shows a sample node, with doors opening on the pathways leading to the related artworks. The interaction metaphor underlying the navigation is "finding one's way": here, however, the user does not simply gain the exit, but the creation of a personal path in the artworks' meaning, represented by a virtual "red thread". In order to make the experience more engaging, when the navigation begins, the user is given a target node. When the user reaches the target, or when she/he decides to exit from the labyrinth, the session ends and the user is shown the statistics about the path she/he has made: elapsed time, number of visited nodes, backtracking, etc.

The user is free to explore the labyrinth, going back to previous locations and clicking on the controls posited in middle of the nodes to receive information about the artworks they contain: depending of the media type of the resource associated with the artwork, an image is displayed, a video is played, etc. A short description of the artwork, with title, date and creator, is also provided. The navigation is inspired by the paradigm of constrained navigation [15], with the aim of making it usable also for non expert users of 3D applications. The user moves in the virtual space by clicking on small circles posited on the floor (in front of the doors leading to the pathways and along the pathways). A small console, situated in the lower part of the screen, shows the list the artworks visited by the user. By clicking on an artwork in the list, the user is brought back to the node containing it. The console also contains buttons for ending the session and turning off the sound.

The glue among the heritage objects stored in the repository is provided by the notion of "archetype", which represents the content of the objects in terms of the archetypal stories they refer to. For example, the archetype of the "labyrinth" can be employed to track the connection between a Greek vase representing the character of Theseus, the playbill of a theatrical play inspired by the story of Ariadne, and a statue depicting Ariadne. The conceptual model of the archetype is encoded in an ontology, the Archetype Ontology (AO), based on previous work by [16]. The ontology contains the representation of a set of archetypes and describes how the artworks are related with them via places, stories, objects, etc.

In order to exemplify the user experience in the 3D labyrinth, consider the navigation example represented in Fig. III. The top part of the figure shows a top down view of the portion of the labyrinth visited by the user in the example; the bottom part contains a sequence of screenshots of the labyrinth as the user sees it during the navigation in the $3 \mathrm{D}$ environment. At the beginning of the navigation (step 1), the user is posited in a node that contains the Minotauromachia, a painting by Pablo Picasso. The initial node is surrounded by a set of doors that represent the semantic relations connecting this artwork with other artworks; each door is labeled after the type of relation it represents: "character" (same character), "story" (same story), "object" (same object), "location" (same location). From the node containing the Minotauromachia, the user chooses the door corresponding to the character relation (step 2): since the are many artworks referring to the characters displayed in the painting, the user is brought to an empty node, whose function is to present to the user the available artworks. Here, the doors are labeled after the titles of the single artworks that are in the chosen relation with the previous node. The user chooses the door surmounted by the title "Teseo uccide il Minotauro" (Theseus killing the Minotaur"), thus entering a pathway that leads her/him to the node which contains the artwork with the same title, an ancient Greek vase (step 3). Here, taking the door labeled as "story", the user gets to a node that contains a painting depicting a different episode of the story of Theseus, an Italian Renaissance painting entitled "Ariadne and the thread".

\section{REAL TIME GENERATION OF THE 3D LABYRINTH}

An important requirement for the design of the Labyrinth $3 \mathrm{D}$ system is given by the possibility of adapting the ontologyto-3D visualization framework to changing, heterogeneous repositories of cultural heritage objects. In order to make the system flexible and scalable, the 3D labyrinth is created during the navigation, node by node, depending on the choices of the user. By doing so, the content of the repository can be arbitrarily changed at any time - provided that the description of items is updated in the ontology. In addition, this solution 


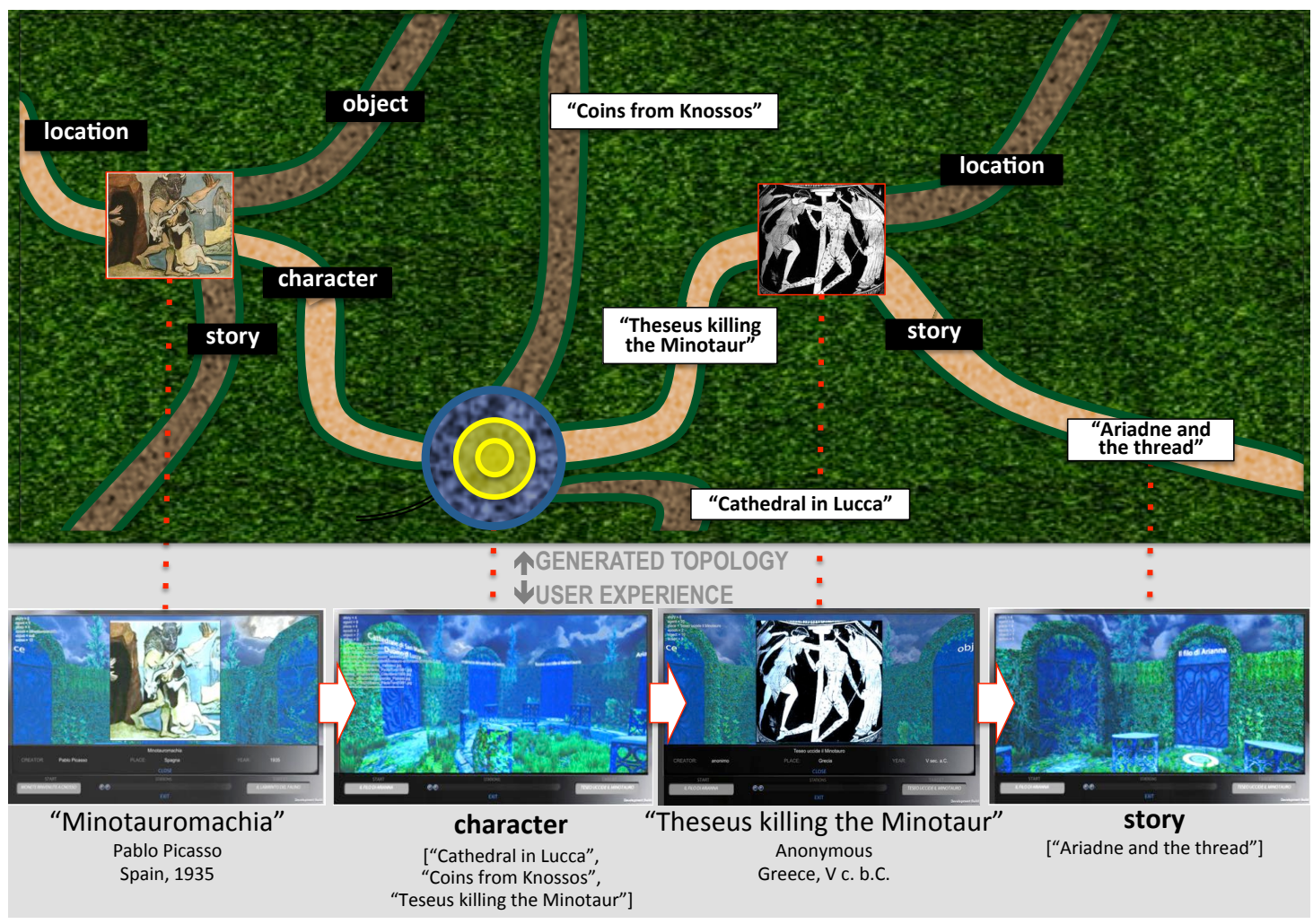

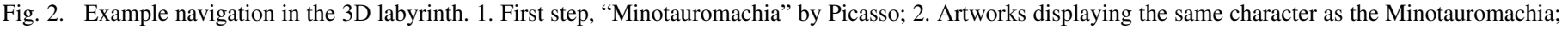
3. "Theseus killing the Minotaur", Greek vase; 4. same story as the Greek vase, "Ariadne and the thread".

avoid the cost of generating the entire labyrinth in one shot, limiting the set of nodes and pathways to be generated to the ones actually visited by the user.

\section{A. System Architecture}

The real time generation relies on a client server architecture, with the 3D client querying the ontology server to get the information needed to generate and render the next portion of the labyrinth. The AO ontology is stored in an ontology server. In the current implementation, the server is provided by Owlim system. ${ }^{2}$ The ontology contains the description of the items in the repository (a set of artworks) and of the relations over them. The information about the artworks and their relations is dynamically extracted from the ontology via SPARQL queries and made available to the visualization client, which manages the $3 \mathrm{D}$ environment.

Each time the user selects a door leading to a new artwork, the 3D client queries the ontology server to get information about the chosen artwork, including the list of artworks it is connected to. A new node for the chosen artwork is then generated and rendered on the fly in the 3D client, along with the set of pathways that connect it with the related artworks retrieved from the ontology.

When the session starts, the visualization client queries the ontology to get the list of the available archetypes. The user chooses an archetype and the client invokes an initialization

\footnotetext{
${ }^{2}$ http://www.ontotext.com/products/ontotext-graphdb-owlim-new-2/
}

command that generates a random pair of artworks (respectively, the start node and target node).

During the session, each time the user selects a door, a loop is repeated that generates the next portion of the labyrinth, until either the user reaches the target node or she/he exits the labyrinth. In practice, the server queries the ontology to extract the semantic relations of the chosen artwork (i.e., the artwork that is the destination of the chosen door) with the other artworks, necessary to build the portion of the labyrinth the user is about to reach. The query executed on the ontology returns the information about the artwork (such as creator, title, etc.) and the list of the semantic relations (such as characterbased, story-based, location-based relations, etc.) that connect it to the other artworks.

The client receives from the ontology server an XML document describing the artwork and its semantic relations with the other artworks, divided by relation type (agent, story, etc.). For each relation type found for the artwork, the visualization client generates the corresponding pathway, according to the following rules:

- 1 to 1 relation: if the relation type connects the chosen artwork with a single artwork, a node is created with the artwork in it, and a pathway is added from the chosen artwork to this node (it is the case of step 4 in Fig. III).

- 1 to many relation: if the relation type connects the chosen artwork with multiple artworks, an empty node is created and a pathway is added from the chosen 
artwork to the empty node (it is the case of step 2 in Fig. III). Then, for each artwork, a node is created with the artwork in it and a pathway is added from the empty node to the new node.

The session ends when the user either reaches the target node or clicks on the exit button in the console.

\section{B. $3 D$ implementation}

The visual layout of the environment is inspired to the classical hedge maze, with clearings and pathways connecting them. The architectural elements of the environment are intended to remind of some distant but not well specified past, so as to create a neutral environment suitable for modern and ancient artworks. The clearings (i.e., the nodes of the labyrinth) contain a low circular balustrade in the middle, open in several points, so as to invite the user to step into the inner part of the area. Each node has a fixed number of doors and corresponding pathways: depending of the number of semantic relations that connect the node with other nodes, some doors may be obstructed (e.g. hidden by greenery). Pathways differ in length and form: some are longer and they bend, so that their end is not visible, in order add some thrill to the experience.

The $3 \mathrm{D}$ realtime engine is provided by Unity 3D. The choice of Unity 3D was determined by previous experience on the platform and the variability/scalability of input/output devices. Unity 3D offers first person gameplay default assets, both concerning camera motion control and mouse tracking motion control. The Unity 3D Engine and the online ontology server dialogue through a specific API, hosted on the server: the choice of an artwork made by the user is passed to the server; when the server returns the class and relations that correspond to the user's choice (the same character, the same author, the same location, etc.), the 3D Engine displays in response the doors as open or closed, all around the current node. The 3D engine also makes the user progress along the pathways in response to the clicks on the navigation controls posited along them.

In order to build the labyrinth on the fly, we built an indexed database of 3D objects to be promptly displayed in real time by the $3 \mathrm{D}$ engine. Thus, we were able to produce several theme variations exploding the number of possible combinations. The standard 3D objects are: the octagonal square for representing the artwork and the relation nodes (3 variants, actually), the open door (3 variants), the closed door ( 2 variants), the textual artwork viewer (1 variant), the pictorial artwork viewer ( 1 variant), the movie viewer (1 variant). The pathways are a 3D object category on their own: they vary in shape in accordance to their length, which is measured in steps (2-3-4 steps, each in three variants). Joined together, steps compose asymmetrical paths, which can also be used backwards, therefore multiplying the possible combinations of subsequent corridors. So, the maze, determined through the user choices, is perceived of never being the same.

\section{CONCLUSION}

In this paper, we described a 3D application that allows the user to navigate a repository of heterogeneous artworks through a physical maze of connections that represent the semantic relation among them.
The preliminary evaluation reported in [17] shows a good acceptance of the system. The architecture of the system provides the basis for developing applications that rely on the same framework, taking advantage of the full range of devices that support real time $3 \mathrm{D}$ to develop innovative cultural heritage applications.

\section{REFERENCES}

[1] E. Hyvönen, "Semantic portals for cultural heritage," in Handbook on ontologies. Springer, 2009, pp. 757-778.

[2] C. Van Den Akker, S. Legêne, M. Van Erp, L. Aroyo, R. Segers, L. van Der Meij, J. Van Ossenbruggen, G. Schreiber, B. Wielinga, J. Oomen et al., "Digital hermeneutics: Agora and the online understanding of cultural heritage," in Proceedings of the 3rd International Web Science Conference. ACM, 2011, p. 10.

[3] P. Mulholland, A. Wolff, and T. Collins, "Curate and storyspace: an ontology and web-based environment for describing curatorial narratives," in The Semantic Web: Research and Applications. Springer, 2012, pp. 748-762.

[4] M. M. Hall, "From searching to using: Making sense of digital cultural heritage collections," in Proc. of "The Search is Over!" Exploring Cultural Collections with Visualization, 2014.

[5] R. Villa, P. Clough, M. M. Hall, and S. Rutter, "Search or browse? casual information access to a cultural heritage collection." in EuroHCIR, 2013, pp. 19-22.

[6] C. Ziemkiewicz and R. Kosara, "Embedding information visualization within visual representation," in Advances in Information and Intelligent Systems. Springer, 2009, pp. 307-326.

[7] C. P. Lim, D. Nonis, and J. Hedberg, "Gaming in a 3d multiuser virtual environment: Engaging students in science lessons," British Journal of Educational Technology, vol. 37, no. 2, pp. 211-231, 2006.

[8] L. P. Rieber and D. Noah, "Games, simulations, and visual metaphors in education: antagonism between enjoyment and learning," Educational Media International, vol. 45, no. 2, pp. 77-92, 2008.

[9] K. Dylla, B. Frischer, P. Mueller, A. Ulmer, and S. Haegler, "Rome reborn 2.0: A case study of virtual city reconstruction using procedural modeling techniques," Computer Graphics World, vol. 16, p. 25, 2008.

[10] V. Lombardo, A. Valle, J. Fitch, K. Tazelaar, S. Weinzierl, and W. Borczyk, "A virtual-reality reconstruction of poeme electronique based on philological research," Computer Music Journal, vol. 33, no. 2, pp. 2447, 2009.

[11] H. Rua and P. Alvito, "Living the past: $3 \mathrm{~d}$ models, virtual reality and game engines as tools for supporting archaeology and the reconstruction of cultural heritage-the case-study of the roman $\mathrm{i}_{i}$ villa $\mathrm{a}_{i} / \mathrm{i}_{i}$ of casal de freiria," Journal of Archaeological Science, vol. 38, no. 12, pp. 32963308, 2011.

[12] N. Smith, K. Knabb, C. Defanti, P. Weber, J. Schulze, A. Prudhomme, F. Kuester, T. Levy, and T. Defanti, "Artifactvis2: Managing real-time archaeological data in immersive 3d environments," in Digital Heritage International Congress. The Eurographics Association, 2013, pp. 363370.

[13] H. Kern and J. Saward, Through the Labyrinth: designs and meanings over 5000 years. Prestel New York, 2000.

[14] P. Rosenstiehl, “Labirinto," Enciclopédia Einaudi, vol. 13, pp. 247-273, 1988.

[15] S. Burigat and L. Chittaro, "Navigation in $3 d$ virtual environments: Effects of user experience and location-pointing navigation aids," International Journal of Human-Computer Studies, vol. 65, no. 11, pp. 945-958, 2007.

[16] R. Damiano and A. Lieto, "Ontological representations of narratives: a case study on stories and actions," in 2013 Workshop on Computational Models of Narrative, CMN 2013, August 4-6, 2013, Hamburg, Germany, ser. OASICS, M. A. Finlayson, B. Fisseni, B. Löwe, and J. C. Meister, Eds., vol. 32. Schloss Dagstuhl Leibniz-Zentrum fuer Informatik, 2013, pp. 76-93. [Online]. Available: http://dx.doi.org/10.4230/OASIcs.CMN.2013.76

[17] R. Damiano, V. Lombardo, and A. Lieto, "Visual metaphors for semantic cultural heritage," in Proc. of Intetain 2015. EAI, 2015. 